\title{
Damage Propagation in Vibrating Masonry Elements using a Nonlocal Description
}

\author{
J. Toti ${ }^{1}$, V. Gattulli ${ }^{1}$ and E. Sacco ${ }^{2}$ \\ ${ }^{1}$ Department of Civil, Construction-Architectural \\ and Environmental Engineering, University of L'Aquila, Italy \\ ${ }^{2}$ Department of Civil and Mechanical Engineering \\ University of Cassino and Southern Lazio, Italy
}

\begin{abstract}
In this paper, damage propagation in vibrating masonry structures is investigated using finite element analyses. A nonlocal damage-plastic constitutive law is adopted to model the macroscopic behavior of the masonry material. In particular, the material model is able to consider different softening laws in tension and in compression, plastic strains, stiffness recovery and loss as a result of crack closure and reopening. The latter effect is an unavoidable feature for realistically reproducing hysteretic cycles caused by harmonic dynamic loadings. Numerical examples concerning different masonry structures demonstrate the applicability of the computational tool developed for reproducing the dynamic response and the damage path evolution.
\end{abstract}

Keywords: dynamic analysis, masonry, nonlocal damage, finite element method.

\section{Introduction}

Cohesive structures, such as masonry or concrete structures constituting a large portion of existing building around the world. In the last forty years, an enormous growth in the development of numerical tools for structural analysis has been achieved. The development of dynamic inelastic analyses became an important tool for the evaluation of the structural behavior of cohesive structures under earthquakes. Computational analysis of cohesive structures subjected to dynamic or cyclic loadings requires realistic stress-strain material models to reproduce realistically the observed behavior. Research efforts on the cyclic response of cohesive material aim at providing an efficient model capable of predicting all the hysteretic characteristics of the material in cyclic loading. Common models able to reproduce the dissipative cycles are based on damage mechanics, plasticity theory and coupling of both. 
Mathematical and numerical models of cohesive material failure must correctly reflect the energy dissipated in the fracture process zone. This is not the case if the stress-strain laws with softening is used within the standard continuum theory. Numerical results obtained with such models suffer from pathological sensitivity to the spatial discretization, e.g. to the size of finite elements. Upon mesh refinement, the energy dissipated by the numerical model decreases and tends to extremely low values, sometimes even to zero. As remedy, the regularized models, based on nonlocal continuum approaches [1-3] can be adopted.

Indeed, the presence of damage or of other inelastic phenomena modifies the overall structural dynamic response and the damage propagation potentially interacts dynamically with the element vibrations; in particular, changes in its behavior are associated to the decay of the mechanical properties of the system [4]. Based on these considerations, many studies have been devoted to use the variations in the dynamic behavior to detect structural damage. Particular attention has been focused on the use of frequencies only, on account of simplicity of measuring them and, therefore, their experimental reliability. In this framework, dynamic analyses of damaged structures have been performed in [5-6] with the aim to detect the damage state of the structure.

In the present work, dynamic finite element analyses of large scale masonry structures are developed. The constitutive material model proposed in [3] and extended for dynamic analyses in [6] is used to reproduce the main hysteretic characteristics of the masonry material under harmonic loadings, such as damage, inelastic strains, unilateral phenomena. The proposed formulation is implemented as constitutive model for two-dimensional plane stress four node quadrilateral elements in a recent version of FEAP code. The second order equations of motion are solved adopting implicit Newmark time integration scheme. The validation and the dynamic performances of the proposed model are demonstrated by numerical examples concerning the analysis of existing masonry structures.

\section{Equations of motion}

A body of volume $\Omega$ subjected to a motion is considered; the weak form of the linear momentum balance, or virtual work expression, takes the following form:

$$
\int_{\Omega} \boldsymbol{\sigma} \cdot \delta \boldsymbol{\varepsilon} d \Omega+\int_{\Omega} \rho \mathbf{a} \cdot \delta \mathbf{u} d \Omega-\int_{\Omega} \mathbf{b} \cdot \delta \mathbf{u} d \Omega-\int_{\partial \Omega} \mathbf{t} \cdot \delta \mathbf{u} d \Omega=0
$$

where $\boldsymbol{\sigma}$ is the stress tensor, $\rho$ is the mass density, $\mathbf{a}$ is the acceleration, $\mathbf{b}$ is the body force, $\mathbf{t}$ is the traction force acting on $\partial \Omega, \delta \boldsymbol{\varepsilon}$ is a strain field compatible with the admissible displacement field $\delta \mathbf{u}$.

The body $\Omega$ is discretized into finite elements and a suitable displacement interpolation is introduced into the virtual work expression, which leads to the ordinary differential system of equations of motion:

$$
\mathbf{M} \ddot{\mathbf{u}}+\mathbf{f}^{\text {int }}(\mathbf{u})=\mathbf{f}^{\text {ext }}
$$


where $\mathbf{u}$ is the nodal displacement vector and $\ddot{\mathbf{u}}$ the nodal acceleration vector, in which dots denote the derivative with respect to time and

$$
\begin{aligned}
\mathbf{M} & =\sum_{i=1}^{n} \int_{\Omega_{i}} \rho \mathbf{N}^{T} \mathbf{N} d \Omega \\
\mathbf{f}^{\text {int }} & =\sum_{i=1}^{n} \int_{\Omega_{i}} \mathbf{B}^{T} \boldsymbol{\sigma} d \Omega \\
\mathbf{f}^{\text {ext }} & =\sum_{i=1}^{n}\left(\int_{\Omega_{i}} \mathbf{N}^{T} \mathbf{b} d \Omega+\int_{\partial \Omega_{i}} \mathbf{N}^{T} \mathbf{t} \partial \Omega\right)
\end{aligned}
$$

with $\mathbf{M}$ the mass matrix, $\mathbf{f}^{\text {int }}$ the internal forces vector, and $\mathbf{f}_{e x t}$ the external force acting upon the system. The matrices $\mathbf{N}$ and $\mathbf{B}$ contain the conventional element interpolation functions and its derivatives, respectively.

A nonlocal damage-plastic model recently proposed in [3] is adopted herein to define the stress $\boldsymbol{\sigma}$ in the material point of the examined structure. The model is able to account for the tensile and compressive damage, the accumulation of irreversible strains and the unilateral phenomenon, i.e. the stiffness recovery and loss due to crack closure and reopening.

The stress-strain constitutive relationship is given by the following expression:

with

$$
\boldsymbol{\sigma}=\overline{\boldsymbol{\sigma}}\left[\left(1-D_{t}\right) H\left(J_{1}^{e}\right)+\left(1-D_{c}\right)\left(1-H\left(J_{1}^{e}\right)\right)\right]
$$

$$
\overline{\boldsymbol{\sigma}}=\mathbf{C}(\boldsymbol{\varepsilon}-\boldsymbol{\pi})=\mathbf{C e}
$$

where $\overline{\boldsymbol{\sigma}}$ are the stress tensor and effective stress tensor, respectively; $\boldsymbol{\varepsilon}, \boldsymbol{\pi}$ and $\mathbf{e}$ are the total strain, the plastic strain and elastic strain, respectively; $\mathbf{C}$ is the fourthorder constitutive tensor; $D_{t}$ and $D_{c}$ are two damage variables which describe the stiffness degradation of the masonry in tension and compression, respectively; $J_{1}^{e}=\operatorname{tr}(\mathbf{e})$ is the first invariant of the elastic strain; $H(x)$ denotes the Heaviside function, $H(x)=1$ if $x \geq 0$, otherwise $H(x)=0$.

The evolution of the plastic strain is described by introducing a yield function representing a branch of a modified hyperbola and the loading-unloading conditions in the Kuhn-Tucker form:

$$
\begin{gathered}
f_{Y}(\overline{\boldsymbol{\sigma}})=A-\left(\bar{\sigma}_{1}-\bar{\sigma}_{Y}\right)\left(\bar{\sigma}_{2}-\bar{\sigma}_{Y}\right)+B\left[\left\langle\bar{\sigma}_{1}-\bar{\sigma}_{Y}\right\rangle_{-}^{2}+\left\langle\bar{\sigma}_{2}-\bar{\sigma}_{Y}\right\rangle_{-}^{2}\right] \leq 0 \\
\dot{\boldsymbol{\pi}}=\dot{\lambda} \frac{\partial f_{Y}}{\partial \overline{\boldsymbol{\sigma}}} \quad \dot{\lambda} \geq 0, \quad f_{Y}(\overline{\boldsymbol{\sigma}}) \leq 0, \quad \dot{\lambda} f_{Y}(\overline{\boldsymbol{\sigma}})=0
\end{gathered}
$$

where $\bar{\sigma}_{1}$ and $\bar{\sigma}_{2}$ are the principal values of the effective stress tensor $\overline{\boldsymbol{\sigma}}$; the bracket symbol $\langle.\rangle_{-}$denotes the negative part of the number; $\bar{\sigma}_{Y}$ is the uniaxial compressive strength of the concrete related to the hyperbola asymptotes $\sigma_{Y}$ through the 
expression $\bar{\sigma}_{Y}=\left(A+\sigma_{Y}{ }^{2}\right) / \sigma_{Y} ; A$ and $B$ are parameters governing the shape of the yield function; in the following analyses, it is always assumed $A=0.1 \mathrm{~N}^{2} / \mathrm{mm}^{4}$ and $B=1 ; \dot{\lambda}$ is the plastic multiplier.

As a damage softening constitutive law is introduced, the localization of the strain and damage variables could occur. In order to overcome this pathological problem, to account for the correct size of the localization zone and, also, to avoid strong mesh sensitivity on the numerical results in finite element analyses, a nonlocal constitutive law is considered both for compressive and tensile damages.

In particular, the evolution of the compressive damage $D_{c}$ is combined with the development of the plastic strain thought the following cubic relationship:

$$
D_{c}=\max _{\text {history }}\left\{0, \min \left\{1, \tilde{D}_{c}\right\}\right\} \quad \text { with } \quad \tilde{D}_{c}=-\frac{2}{\kappa_{u}^{3}} \bar{\kappa}^{3}+\frac{3}{\kappa_{u}^{2}} \bar{\kappa}^{2}
$$

where $\kappa_{u}$ is the final accumulated plastic strain associated with the compressive damage $D_{c}=1 ; \bar{\kappa}$ is the nonlocal counterpart of the accumulated plastic strain given by the following expression:

$$
\bar{\kappa}(\mathbf{x})=\frac{1}{\int_{\Omega} \psi_{c}(\mathbf{x}, \mathbf{y}) d \Omega} \int_{\Omega} \psi_{c}(\mathbf{x}, \mathbf{y}) \kappa(\mathbf{y}) d \Omega
$$

being $\kappa=\int_{0}^{t}\|\dot{\boldsymbol{\pi}}\| d t$ the local value of the accumulated plastic strain; $\psi_{c}(\mathbf{x}, \mathbf{y})=\left\langle 1-\|\mathbf{x}-\mathbf{y}\|^{2} / R_{c}^{2}\right\rangle_{+}$the compressive weight function which determines the influence of the point $\mathbf{y}$ on $\mathbf{x}$; the bracket symbol \langle\rangle$_{+}$the positive part of the number; the parameter $2 R_{c}$ defines the compressive characteristic length, which contributes to the definition of nonlocal accumulated plastic strain.

The evolution of the tensile damage parameter $D_{t}$ is governed through the following nonlocal exponential law:

$$
D_{t}=\max _{\text {history }}\left\{0, \min \left\{1, \tilde{D}_{t}\right\}\right\} \quad \text { with } \quad \tilde{D}_{t}=\frac{\bar{\varepsilon}_{e q}-\varepsilon_{0} \exp \left(-k\left(\bar{\varepsilon}_{e q}-\varepsilon_{0}\right)\right)}{\bar{\varepsilon}_{e q}}
$$

where $\varepsilon_{0}$ is a material parameter indicating damage threshold strain; $\bar{\varepsilon}_{e q}$ is the nonlocal equivalent strain defined as:

$$
\bar{\varepsilon}_{e q}(\mathbf{x})=\frac{1}{\int_{\Omega} \psi_{t}(\mathbf{x}, \mathbf{y}) d \Omega} \int_{\Omega} \psi_{t}(\mathbf{x}, \mathbf{y}) \varepsilon_{e q}(\mathbf{y}) d \Omega
$$

with $\varepsilon_{e q}=\sqrt{\left\langle e_{1}\right\rangle_{+}^{2}+\left\langle e_{2}\right\rangle_{+}^{2}}$ the equivalent elastic strain; $e_{1}$ and $e_{2}$ are the principal elastic strains; $\psi_{t}(\mathbf{x}, \mathbf{y})=\left\langle 1-\|\mathbf{x}-\mathbf{y}\|^{2} / R_{t}^{2}\right\rangle_{+}$defines the tensile weight function, with $2 R_{t}$ the tensile characteristic length, which contributes to the definition of nonlocal 
equivalent strain. Finally, the condition that the damage in compression must be lower than the one in tension is enforced $D_{t} \geq D_{c}$.

The constitutive law defined in equation (4), for particular strain paths and damage conditions can be lead to discontinuities in the response of the material point. Indeed, for an isotropic cohesive material, characterized by the shear modulus $G$, subjected to $J_{1}$ history and to a constant elastic shear strain $e_{x y}$, the shear stress is $\sigma_{x y}=2 G e_{x y}\left[\left(1-D_{t}\right) H\left(J_{1}^{e}\right)+\left(1-D_{c}\right)\left(1-H\left(J_{1}^{e}\right)\right)\right]$. If $D_{t}>D_{c}$ the value of shear stress $\sigma_{x y}$ undergoes a sudden jump, when $J_{1}^{e}$ changes from positive to negative value or vice-versa. While it can be considered realistic to have a stiffer shear response when the material point is subjected to volumetric contraction with respect to the case of volumetric expansion, because of the positive effect of the friction in compression, this strong discontinuity of response of the material point can be considered undesirable from both a physical and a mathematical point of view. For this reason, a regularized form of the Heaviside function can be adopted with the aim to avoid annoying jumps in the response; in particular, the following regularized form is considered for the Heaviside function:

$$
H\left(J_{1}^{e}\right)=\frac{1}{e^{-J_{1}^{e} / h}}
$$

where $\mathrm{h}$ is a small parameter governing the regularization effect $(\mathrm{h}=0.0001 \div 0.001)$. From the set of equations (2), only the equations related to the free-coordinates $\tilde{\mathbf{u}}$ can be discerned and, taking into account the boundary conditions on the restrainedcoordinates $\hat{\mathbf{u}}$, they are rewritten in following form:

$$
\tilde{\mathbf{M}} \ddot{\tilde{\mathbf{u}}}+\tilde{\mathbf{f}}^{\mathrm{int}}(\tilde{\mathbf{u}})=\tilde{\mathbf{f}}^{e x t}-\tilde{\mathbf{M}} \ddot{u}_{g}
$$

where $\tilde{\mathbf{M}}, \tilde{\mathbf{f}}^{\text {int }}$ and $\tilde{\mathbf{f}}^{\text {ext }}$ are the restrictions of $\mathbf{M}, \mathbf{f}^{\text {int }}$ and $\mathbf{f}^{\text {ext }}$ to the degrees of freedom $\tilde{\mathbf{u}}$; moreover, $u_{g}$ is an imposed displacement time-history at the ground in the direction described by the allocation vector $\mathbf{r}$ and $\ddot{u}_{g}$ is its second time derivative, i.e. the ground acceleration.

The proposed nonlocal material model is implemented as a constitutive model for two-dimensional four-node quadrilateral elements usable for direct integration analyses of the equation (13) in a research version of the finite element code FEAP.

\section{Dynamic response and damage propagation}

\subsection{Selected masonry elements}

The dynamic behaviour and the damage propagation are investigated for large scale masonry structures. Specifically, two case studies are considered: a vertical structural member (VM) and a curved structural member (CM). The first masonry element is existing and belongs to the Basilica S. Maria di Collemaggio, the most celebrated medieval church in the Italian Region of Abruzzo, located in the town of 
L'Aquila. The analysis aims to demonstrate the ability of the procedure to reproduce the final crack pattern on the columns as observed immediately after the 2009 L'Aquila earthquake [8]. The second one is the masonry arch studied during the experimental campaign presented in [6]. The geometry of the examined mechanical systems are reported in Figure 1. The vertical member, illustrated in Figure 1a, is a portion of one of the masonry nave walls of the Basilica and it is composed of the central column and two half ogival arches above it.

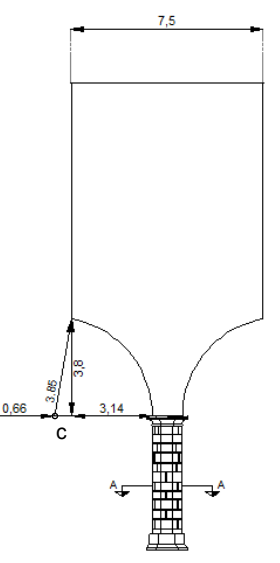

a)
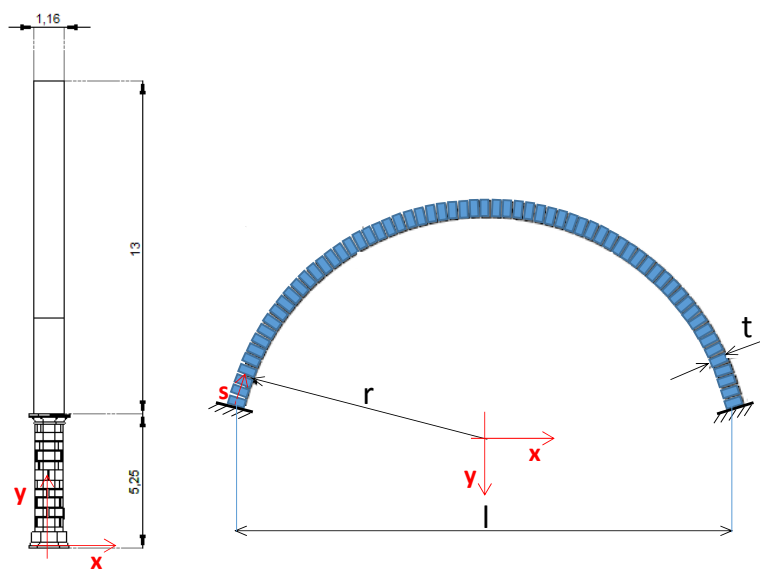

b)

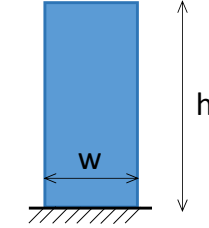

Figure 1: Geometry: a) vertical member (units in metres); b) curved member.

The cross section of the column is octagonal and each of the eight sides measuring 0.5 meters wide. The masonry arch belongs to an experimental setup apparatus and it is built with standard clay bricks with size $100 \times 50 \times 25 \mathrm{~mm}$. The geometrical data of the tested circular arch, schematically reported in Figure $1 \mathrm{~b}$, are the following: internal radius $r=0.77 \mathrm{~m}$; width $w=0.45 \mathrm{~m}$; thickness $t=0.05 \mathrm{~m}$; height $h=0.59 \mathrm{~m}$; span $l=1.50 \mathrm{~m}$.

\subsection{Modeling}

A two-dimensional finite element model is developed in the $x-y$ plane according to the introduced reference system represented in Figure 1 for each of the two studied structures. The discretization of the two mechanical systems assures a satisfactory numerical result, particularly with reference to the initial evaluation of the elastic modal analysis. Indeed, considering refined meshes characterized by a higher number of finite elements, the relative error in the first fundamental modal frequencies is negligible. In particular, the finite element model together with the first two frequencies and modal shapes of the vertical member are depicted in Figure 2a,b,c, respectively, while in Figure 2d,e,f are reported the same entities for the studied arch.

The mechanical properties of the two masonry structures are contained in Table 1. The model parameters adopted for the vertical structure are set on the basis of global and local tests summarized in [8] which are also object of a permanent monitoring using a wireless sensor network $[9,10]$, while those assumed for the 
masonry arch are defined through the development of an inverse procedure based on the results of the dynamic and static tests carried out in [6].

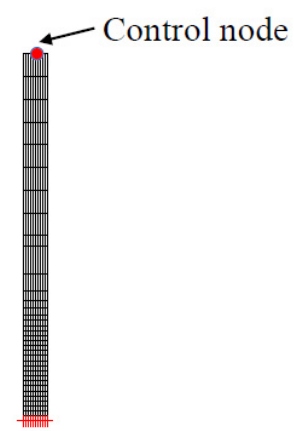

a)

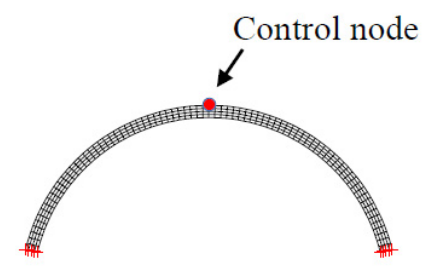

d)

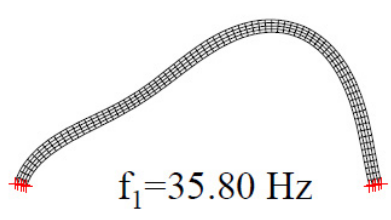

e)

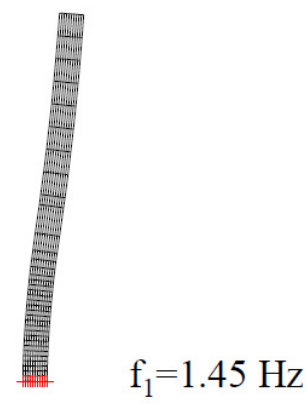

b)

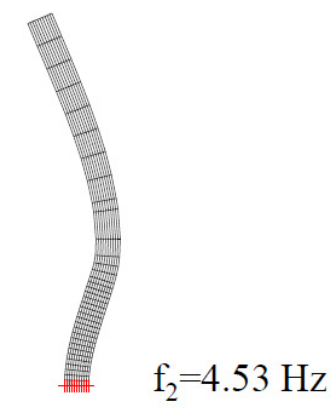

c)

Figure 2: Vertical member, a) mesh, b) Mode 1, c) Mode 2; curved member, d) mesh, e) Mode 1, f) Mode 2

\begin{tabular}{|c|c|c|c|c|c|c|c|c|c|}
\hline & & $\begin{array}{c}E \\
{[\mathrm{MPa}]}\end{array}$ & $v$ & $\varepsilon_{0}$ & $\begin{array}{c}\sigma_{Y} \\
{[\mathrm{MPa}]}\end{array}$ & $\kappa_{u}$ & $k$ & $\begin{array}{c}R \\
{[\mathrm{~m}]}\end{array}$ & $\begin{array}{c}\rho \\
{\left[\mathrm{kN} / \mathrm{m}^{3}\right]}\end{array}$ \\
\hline \multirow{2}{*}{ V.M } & $\begin{array}{c}\text { Ogival } \\
\text { Arch }\end{array}$ & 2500 & 0.2 & $\begin{array}{c}1.2 \mathrm{e}- \\
004\end{array}$ & -3 & $\begin{array}{c}- \\
0.07\end{array}$ & 200 & 0.4 & 21.35 \\
\hline & Column & 20400 & 0.2 & $5 e-005$ & -3 & $0 . \overline{07}$ & 200 & 0.4 & 17 \\
\hline C.M & & 4000 & 0.2 & $\begin{array}{c}3.5 \mathrm{e}- \\
005\end{array}$ & -1 & 0.07 & 500 & 0.03 & 20 \\
\hline
\end{tabular}

Table 1: Material parameters adopted for the vertical member (VM) and for the curved member $(\mathrm{CM})$.

\subsection{Numerical results}

Damage propagation induced by synchronous motion applied to all nodes of base of the two mechanical systems is analysed. The imposed base motion is a sinusoidal function of time $u_{g}(t)=u_{0} \sin \left(2 \pi f_{0} t\right)$, defined by two parameters $u_{0}$ and $f_{0}$ indicating the frequency and the displacement amplitude of the harmonic imposed motion at the ground, respectively. 

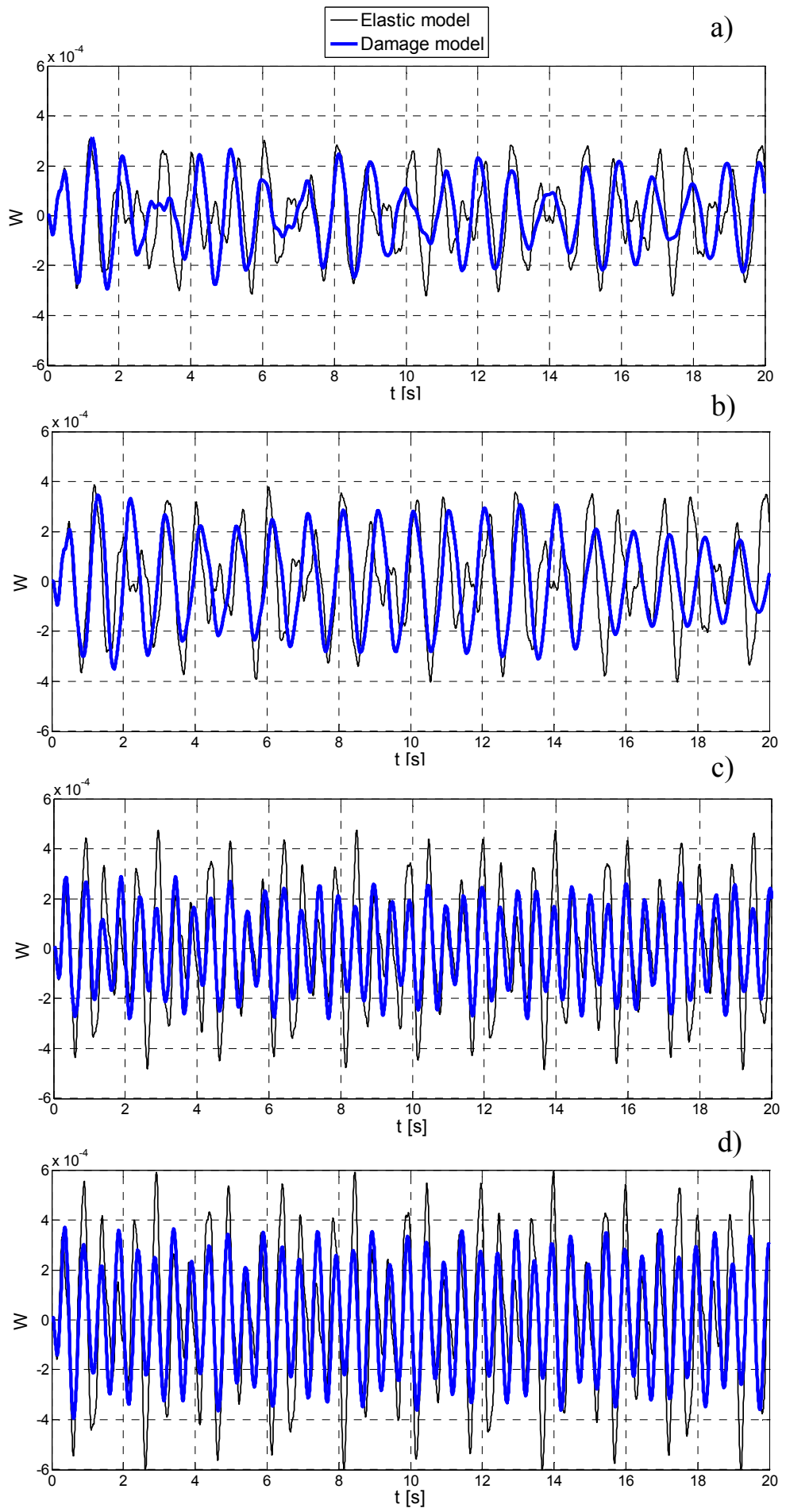

Figure 3: Time history of the normalized vertical displacement $\mathrm{W}$ of the control node considered for VM, $\eta=0.7$ a) $u_{0}=4 \mathrm{~mm}$; b) $u_{0}=5 \mathrm{~mm} ; \eta=1.3 \mathrm{c}$ ) $u_{0}=4 \mathrm{~mm}$; d) $u_{0}=5 \mathrm{~mm}$. 


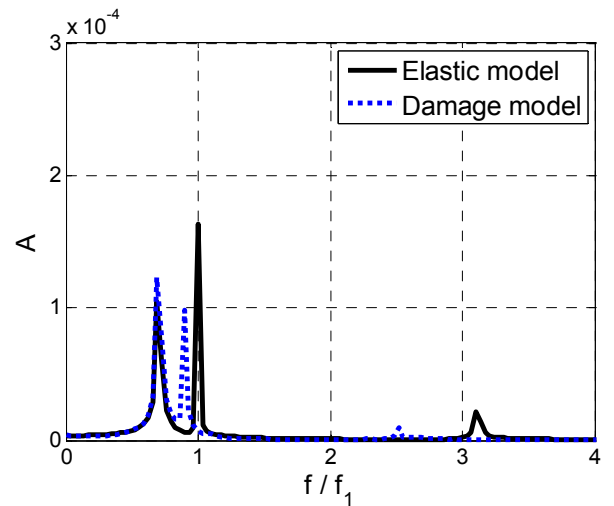

a)

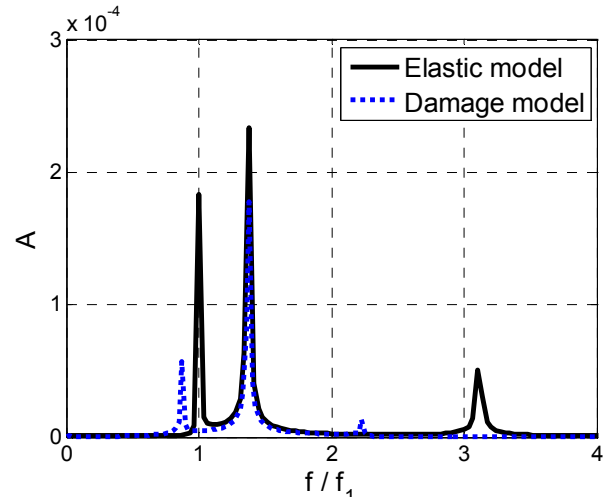

b)
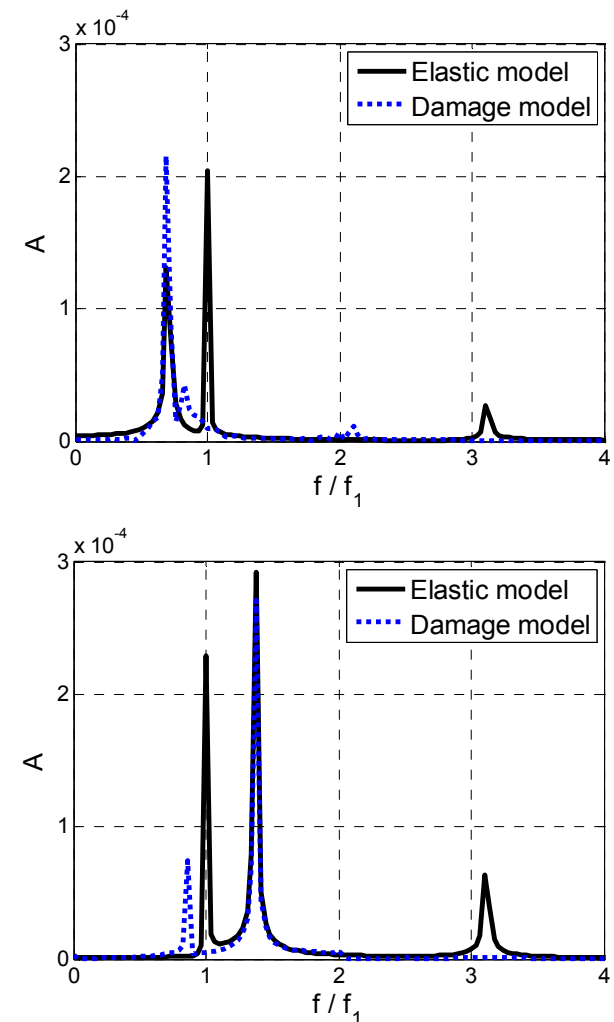

c)

Figure 4: Fourier spectra of the normalized displacement response $\mathrm{W}$ for VM

$$
\begin{aligned}
& \eta=0.7 \text { a) } u_{0}=4 \mathrm{~mm} ; \text { b) } u_{0}=5 \mathrm{~mm} ; \eta=1.3 \text { c) } u_{0}=4 \mathrm{~mm} ; \text { d) } \\
& u_{0}=5 \mathrm{~mm} .
\end{aligned}
$$

Numerical analyses are performed considering the frequency ratio $\eta=f_{0} / f_{1}$ equal to 0.7 and 1.3. For each frequency ratio, two values of the displacement amplitude are considered. Specifically, in the case of the vertical structural member, the displacement amplitudes are assumed equal to 4 and $5 \mathrm{~mm}$, while, for the curved structure, they are set equal to 0.1 and $0.11 \mathrm{~mm}$. The choice of these displacement amplitude values, for the two chosen frequencies ratio, leads to base accelerations, which induce damage states in the examined masonry elements.

For the two masonry elements, the equations of the motion are integrated until the time $20 \mathrm{~s}$ with a time step equal to approximately $1 / 14$ of the second natural period of vibration in order to correctly integrate the first two modes.

The dynamic behavior of the masonry element is studied in term of time histories of a nodal displacement of the finite element discretization. In particular, it is considered:

- for the vertical structural member, a normalized displacement $\mathrm{W}$, defined as the ratio between the horizontal displacement of the center point at the top of the structure and the total height of the element (Figure 2a);

- for the masonry arch, a normalized displacement $\mathrm{V}$, computed as the ratio between the vertical displacement at the extrados of the key-section of the arch and its span (Figure 2c). 

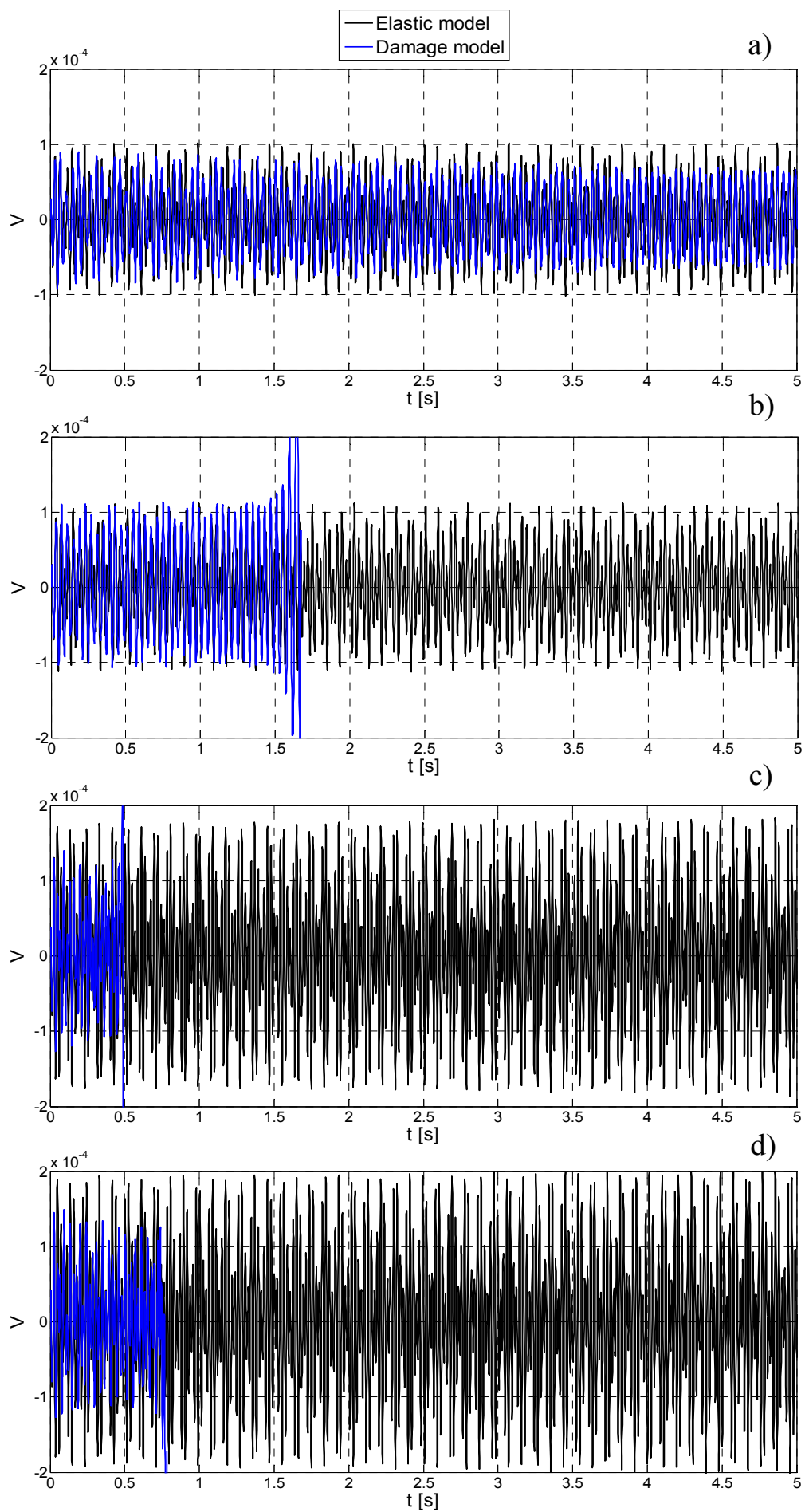

Figure 5: Time history of the normalized vertical displacement $\mathrm{V}$ of the control node considered for the curved structural member, $\eta=0.7$ a) $u_{0}=0.10 \mathrm{~mm} ; \quad$ b) $u_{0}=0.11 \mathrm{~mm} ; \eta=1.3 \quad$ c) $u_{0}=0.10 \mathrm{~mm} ; \quad$ d) $u_{0}=0.11 \mathrm{~mm}$. 

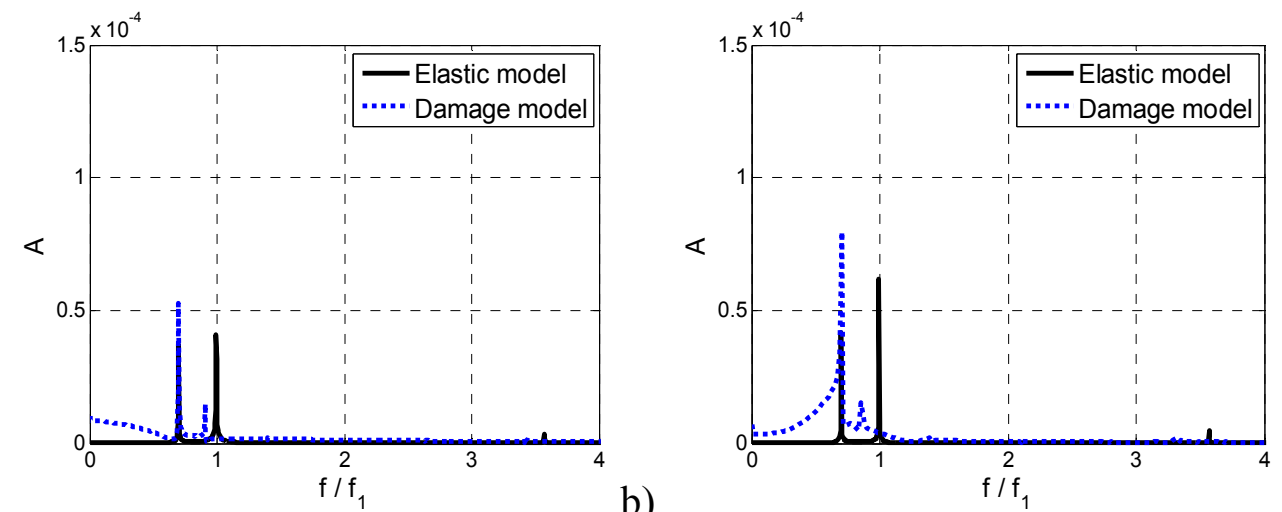

a)

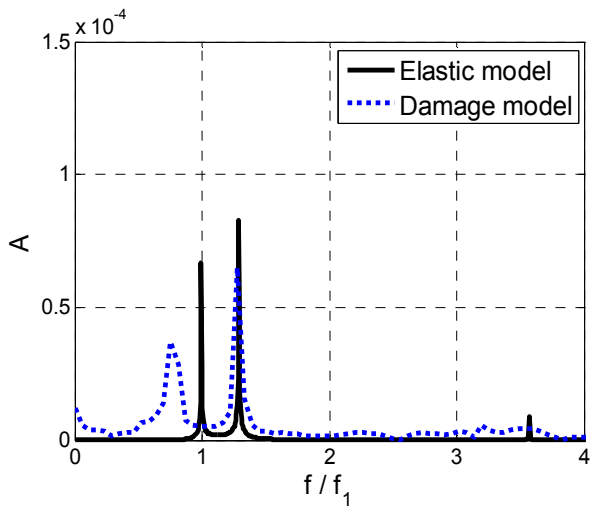

b)

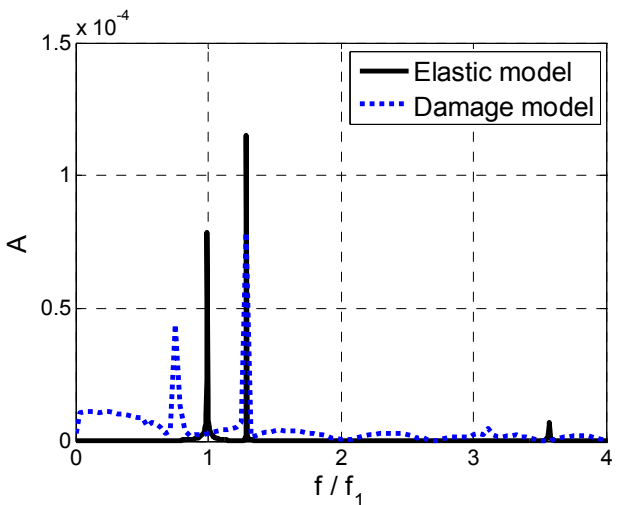

Figure 6: Fourier spectra of the normalized displacement response V: $\eta=0.7$ a)

$$
\begin{aligned}
& u_{0}=0.10 \mathrm{~mm} ; \quad \text { b) } u_{0}=0.11 \mathrm{~mm} ; \quad \eta=1.3 \quad \text { c) } u_{0}=0.10 \mathrm{~mm} \\
& u_{0}=0.11 \mathrm{~mm}
\end{aligned}
$$

Figure 3 and Figure 4 report the displacement responses and Fourier spectra, respectively, obtained in the case of the vertical structural member; Figure 5 and Figure 6 show the numerical results in term of time history of the nodal displacement and spectral analyses determined for the curved structure. In these figures, the comparison between the damage model and the elastic model is also provided. It can be remarked that: the damaging of the structure leads to a decrease of the natural frequencies of the systems; for constant values of $\eta$, the reduction is faster with increasing of the forcing displacement amplitude; the cases with $\eta=0.7$ and higher displacement amplitudes induce an on-going resonance mechanism between the decreasing first natural frequency and the lower forcing frequency, which causes the collapse of the two masonry structures; the masonry arch reaches the failure mechanism before the vertical structural member, because the curved member with respect to the other structural system has higher values of the natural frequencies which lead to the fulfilment of a greater number of cycles; the analysed masonry arch reaches before of $20 \mathrm{~s}$ the collapse condition in the case of large accelerations (i.e. for $\eta=1.3$ ).

Figure 7a-b illustrate the stress-strain curves for the vertical and curved member, respectively, at a Gauss point of a finite element located in correspondence of the base. From the figures, it can possible to observe that: the damage in tension occurs, 
while the damage in compression is not reached; the effect of the unilateral contact, due to stiffness recovery and loss due to crack closure and reopening, is well simulated; in the material point response of the masonry arch a number of hysteretic cycles greater than one occurring in the other structure develops.
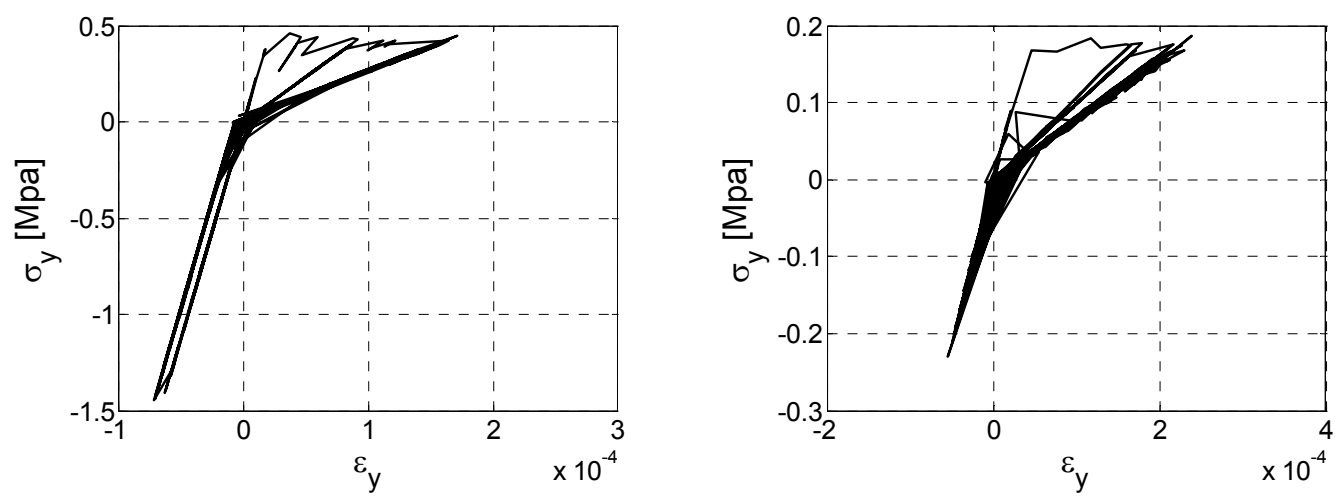

Figure 7: Stress-strain curve at Gauss point of a finite element located at the base: a) for vertical member; b) for curved member.

\section{Conclusions}

A computational tool is developed for the analyses of the dynamic behaviour of large scale masonry elements. A vertical structural member and a simple curved member are considered as case studies. Two-dimensional finite element models of these two structures are developed. A nonlocal plastic damage model is adopted in order to reproduce the cyclic macroscopic behavior of the masonry material. The model considers different softening laws in tension and in compression, plastic strains, stiffness recovery and loss due to crack closure and reopening. Moreover, it is formulated in a nonlocal continuum approach, in which the variables governing the damaging in tension and in compression are defined thorough an integral-type technique.

The dynamic responses of these damaging systems under sinusoidal imposed base motions are investigated; the effect of both the amplitude and the frequency content of the base motion on damage propagation is examined thorough the Fourier spectra. The numerical results show that the damaging of the masonry induces a decrease of the natural frequencies of the system. Moreover, for a forcing frequency lower than the first natural frequency computed in the undamaged condition, a peculiar evolutive phenomenon occurs for the both masonry structures. In particular, the damage produces a reduction of the first natural frequency, which causes an ongoing resonance mechanism. The base motions at forcing frequency higher than the first natural one is accompanied by large accelerations which induce the collapse of the structure only for the case of the masonry arch.

Finally, some examples of local responses demonstrate the ability of the proposed model in reproducing the macroscopic behaviour of the masonry material under cyclic loadings. 


\section{References}

[1] G. Pijaudier - Cabot, Z. Bažant, "Nonlocal Damage Theory", J. Eng. Mech., 113(10), 1512-1533, 1987.

[2] G. Borino, B. Failla, F. Parrinello, "A symmetric nonlocal damage theory", Int. J. Solids Struct, 40, 3621-3645, 2003.

[3] J. Toti, S. Marfia, E. Sacco, "Coupled body interface nonlocal damage model for FRP detachment”, Comput. Method Appl. M., 260, 1-23, 2013.

[4] J. Toti, V. Gattulli, E. Sacco., "Nonlocal damage propagation in the dynamics of masonry structures", Computers \& Structures, submitted, 2014.

[5] U. Andreaus, P. Casini, F., Vestroni, "Nonlinear dynamics of a cracked cantilever beam under harmonic excitation", Int. J. Nonlin. Mech., 42, 566575, 2007.

[6] L.F. Ramos, G. De Roeck, Lourenço P.B., Campos-Costa, A., "Damage identification on arched masonry structures using ambient and random impact vibrations", Engineering Structures 32, 146-162, 2010.

[7] J. Toti, V. Gattulli, E. Sacco, "Damage propagation in a masonry arch subjected to slow cyclic and dynamic loadings", Frattura ed Integrità Strutturale, 29, 166-177, 2014.

[8] V. Gattulli, E. Antonacci, F. Vestroni, "Field observations and failure analysis of the Basilica S. Maria di Collemaggio after the 2009 L'Aquila earthquake", Engineering Failure Analysis, 34, 715-734, 2013.

[9] F. Federici, F. Graziosi, M. Faccio, V. Gattulli, M. Lepidi, F. Potenza, , “An integrated approach to the design of Wireless Sensor Networks for structural health monitoring. International Journal of Distributed Sensor Networks, 2012, 16, 2012.

[10] V. Gattulli, F. Graziosi, Federici, F., Potenza, F., Colarieti, A., M. Lepidi, "Structural health monitoring of the Basilica S. Maria di Collemaggio." in Research and Applications in Structural Engineering, Mechanics and Computation, Proc. 5th International Conference on Structural Engineering, Mechanics and Computation SEMC2013, di Zingoni A. (editore), Cape Town (Sudafrica), Settembre 2013. ISBN: 978-11-38000-61-2. 